\title{
RADIAL VELOCITY OF THE CENTRAL STAR OF NGC 1514
}

\author{
A. Mammano, R. Margoni, M. Perinotto \\ (Asiago Astrophysical Observatory, Italy)
}

We would like to report on some spectroscopic observations made of the central star of the planetary nebula NGC 1514, which we think can support the binary hypothesis now advanced by Kohoutek.

Between 1965 and 1967, 21 spectra (dispersions of 72 and $42 \AA / \mathrm{mm}$ at $\mathrm{H} \gamma$ ) have been obtained with the $122-\mathrm{cm}$ reflector at Asiago. The radial velocity of the central star of NGC 1514 varies, according to our measurements, from $+35 \pm 10 \mathrm{~km} / \mathrm{sec}$ to +95 $\pm 10 \mathrm{~km} / \mathrm{sec}$. Three maxima and three minima can be recognized among the 17 spectra measured so far. On the other hand, the physical association between the star and the nebula is confirmed by the agreement between the radial velocity of the absorption lines of the star and that of the nebular lines which is according to Chopinet (private communication), $70 \pm 18 \mathrm{~km} / \mathrm{sec}$, obtained with a dispersion of $380 \AA / \mathrm{mm}$.

These preliminary results appear to confirm the binary nature for the nucleus of NGC 1514, although further analysis is required to test the possibility that we are confronted with a close binary, and this work is in progress.

\section{DISCUSSION}

Miller: If the radial velocity variation of the star is interpreted as binary motion, what would be the best estimate of the orbital period?

Perinotto: It is not yet possible for us to give an estimate of the period. We need a larger number of spectra than we have. 